Voix et Images

voixetimages

\title{
Entre France et Québec : des lieux, des liens, une voix
}

\section{Monique La Rue}

Volume 13, numéro 1 (37), automne 1987

Suzanne Lamy

URI : https://id.erudit.org/iderudit/200682ar

DOI : https://doi.org/10.7202/200682ar

Aller au sommaire du numéro

\section{Éditeur(s)}

Université du Québec à Montréal

\section{ISSN}

0318-9201 (imprimé)

1705-933X (numérique)

Découvrir la revue

\section{Citer cet article}

La Rue, M. (1987). Entre France et Québec : des lieux, des liens, une voix. Voix et Images, 13(1), 42-45. https://doi.org/10.7202/200682ar d'utilisation que vous pouvez consulter en ligne.

https://apropos.erudit.org/fr/usagers/politique-dutilisation/ 


\title{
Entre France et Québec: des lieux, des liens, une voix
}

\author{
par Monique La Rue, Cégep Édouard-Montpetit
}

Chacun d'entre nous est unique et irremplaçable. Suzanne Lamy occupait, sur la scène littéraire montréalaise, une place tout à fait singulière. Elle fut non seulement l'auteure prolifique de critiques, d'essais et de textes de fiction, mais aussi une animatrice particulièrement dynamique, qui eut le souci de créer et de maintenir des lieux de parole libres et novateurs.

Un des aspects les plus profonds de limpact qu'elle a pu avoir dans notre milieu tient à ce que, tout en étant intimement liée à l'évolution de la littérature d'ici, elle ne se sentit pas pour autant obligée de renier ce que son éducation et sa culture européennes lui avaient transmis. À l'heure où il est de bon ton de valoriser le soi-disant cosmopolitisme de notre culture, au moment où nos réactions xénophobes de naguère semblent aux yeux de plusieurs choses du passé, les Français de Montréal sont peut-être un des groupes envers lesquels les Québécois gardent le plus de méfiance. Suzanne Lamy connaissait parfaitement ce contentieux. Elle a su l'assumer sans le nier et, sans se trahir elle-même, en accepter les contradictions. Elle appréciait la jeunesse et la souplesse de notre monde, en comparaison des barrières sociales, des «conventions» et des codes qu'elle avait senti peser sur sa société d'origine. Mais elle savait aussi garder face aux problèmes du Québec une distance qui, si elle ne fut pas toujours appréciée, me semble avoir été un de ses apports les plus féconds à notre vie littéraire.

Cette position lui donnait ainsi un recul qui la poussait continuellement à faire réfléchir sur le rapport à la langue, ce qui est bien une des définitions de l'écriture. Pour avoir examiné avec elle les articles destinés à la revue Spirale, j'ai pu admirer sa connaissance intime et précise du français qui lui venait, m'avaitelle dit, de sa mère, institutrice. Elle comprenait parfaitement les raisons qui ont pu pousser la langue littéraire québécoise à explorer ce qu'on a appelé le «joual». Elle était aussi profondément sensible aux colorations misogynes de la grammaire française. Ennemie jurée du «bien écrire», elle était la plus éloignée du monde d'une conception puriste ou normative de la langue. Mais elle ne manquait jamais de questionner, avec discrétion, les tendances suicidaires qui risqueraient d'enfermer la langue québécoise dans un ghetto vernaculaire ou patoisant.

Moins que toute autre rationaliste au sens étroit du terme, elle savait apprécier la valeur de l'immotivé dans l'écriture: sa thèse de doctorat portait sur 
Arcane 17. Mais elle ne confondait pas pour autant liberté et laxisme. Son exigence de précision et de rigueur lorsqu'elle avait à accepter ou à travailler les textes des autres ne sont peut-être pas non plus ce qu'elle a fait de plus visible. Mais, outre le fait qu'elle en a effectivement amélioré plus d'un, elle témoigne, dans notre microcosme, d'une détermination et d'un respect des exigences de la pensée qui resteront pour moi un modèle. Et ce, bien au-delà de mon accord ou de mon désaccord avec les idées qu'elle a promues, ou des jugements qu'elle portait, en toute indépendance d'esprit, sans jamais se mentir à elle-même ou aux autres, au risque de déplaire et même de se tromper.

Plus d'une fois elle aura jeté des ponts entre la France et le Québec, ce qui n'arrive pas si souvent qu'on pense. En 1981 elle eut l'idée de saluer, dans un livre dont elle a été l'initiatrice et, en collaboration avec André Roy, l'éditrice dans tous les sens du mot, l'écrivaine Marguerite Duras. $\AA$ ce moment, il faut s'en souvenir, le prix Goncourt et ses avant-signes n'avaient pas commencé à faire de celle-ci la vedette littéraire qu'elle est devenue peu après. Cette initiative, suivie d'un colloque au Centre culturel canadien de Paris en 1982 qui compte parmi les activités les mieux réussies du Centre, fut couronnée de succès, et tout le tirage de Marguerite Duras à Montréal, distribué au Québec et en France, fut vendu. Ceux et celles qui ont collaboré à cette entreprise non dénuée de risques, savent dans quel climat d'amitié, de connivence et de simplicité cela fut fait. Il reste à mes yeux tout à fait singulier que des Québécois aient ainsi eu l'occasion de se réunir autour d'une oeuvre française, et cela juste avant que le grand public international ne lui apporte une reconnaissance définitive.

Nombreux furent les contacts de Suzanne Lamy avec les écrivaines et écrivains européens, sa participation à des colloques québécois ou internationaux. Je crois que cela tient à une qualité qu'elle avait et qui, même si elle n'est pas d'ordre intellectuel ou littéraire, n'est pas si éloignée du travail d'écrivain: c'était une femme qui savait créer des liens. Elle savait garder ses positions dans une discussion, tout en sauvegardant le plus longtemps possible le sens de l'humour. Si elle ne recula pas devant les ruptures qu'elle jugea inévitables, elle ne rompit jamais à la légère. Car elle n'était pas de ceux qui séparent mesquinement les relations professionnelles et l'amitié.

Avec d'elles, elle témoigna de la valeur qu'elle accordait aux amies avec lesquelles elle partageait, bien sûr, ce qu'on a pu appeler la «condition féminine», mais d'abord et surtout un certain rapport au langage. $\grave{A}$ de nombreuses occasions, dans des revues, des forums, des rencontres qu'il serait fastidieux d'énumérer, elle fut invitée à parler des «grandeurs et misères de la critique au féminin», comme le dit le titre d'un de ces colloques auquel elle participa à l'Institut Simone de Beauvoir de l'Université Concordia. Et même quand elle fut lasse d'être associée à ce qu'elle avait contribué à promouvoir, parce qu'elle ne croyait plus pouvoir en renouveler la problématique, elle ne se déchargea jamais de ce sujet. 
Elle avait dit, dans un colloque organisé par la revue Critère, la difficulté et la fascination de la «trans/mission» du savoir à notre époque post-moderniste. Enseignante au Cégep du Vieux-Montréal, elle contribua à la mise sur pied d'un cours sur l'écriture au féminin qui allait inspirer d'autres institutions de même niveau, faire connaître des auteures, mais surtout susciter chez les étudiantes et étudiants qui le suivirent la conscience de la brèche que le «deuxième sexe» a réussi à percer, au vingtième siècle, dans une tradition apparemment inamovible.

Si c'est donc probablement à bon droit qu'on a pu la qualifier de «féministe», Suzanne Lamy ne le fut cependant jamais, à ma connaissance, de façon dogmatique. Ne la vit-on pas, dans Éros au pluriel, se passionner pour l'histoire sulfureuse de Mara1, qu'on ne saurait certes soupçonner d'être une suffragette... Son désir d'un changement pour les femmes s'inscrivait ainsi, bien au-delà du champ politique, dans une attitude fondamentale d'ouverture à ce qu'on a pu appeler «la modernité», dans un rejet viscéral de toutes les scléroses, et dans la défense naturelle de la liberté des individus. S'il est une constante des activités de Suzanne Lamy, c'est bien ce désir de favoriser le changement et de rester «moderne» au sens absolu de ce mot: «qui est du temps de celui qui parle». C'est dans ce sens aussi qu'elle co-anima, avec France Théoret en 1981, une soiréedébat de l'Uneq dont le thème était «La mère, aujourd'hui», dont elle reprit le sujet dans un autre colloque organisé en 1982 par l'Association des professeurs de français du Québec.

C'est également cette recherche des énergies de changement qui l'amena d'emblée à collaborer à Spirale. Si, chronologiquement, elle n'était pas de leur génération, elle partageait avec les gens qui gravitaient autour de cette revue la crainte du folklorisme et le désir de faire avancer les choses. Elle devait même contribuer à sauver la revue, en 1981, lorsque l'équipe fondatrice connut des difficultés financières et une syncope morale dont la tira un regroupement de fortune, composé pendant un temps uniquement de femmes. Avec France Theoret alors, et ensuite à titre de directrice, elle continua à entretenir cet esprit «Spirale» qui était profondément le sien: donner un lieu pour une parole ... intéressée aux productions les plus novatrices du champ culturel 2 . Favorisant tout ce qui pouvait contribuer à susciter ou à éclairer des rapports entre les oeuvres québécoises et les productions ou les problématiques novatrices venant d'ailleurs, elle consolida à sa façon la vocation spécifique de Spirale. Mais elle accepta également, sans compter son temps et son énergie, d'en faire la promotion, de défendre et d'expliciter sur la place publique des positions critiques et éditoriales qui ne firent d'ailleurs pas toujours l'unanimité. En témoigne entre autres un échange de lettres dans le Devoir avec Lise Bissonnette ${ }^{3}$. De Spirale, qui lui tenait tant à coeur, elle sut aussi se retirer, non sans avoir veille à assurer la

1 Mara ou le journal d'une femme soumise, Flammarion, 1979.

2 «Pourquoi Spirale?», Bulletin de l'Association des études canadiennes, vol. 8, no 2 , été 1986 , p. 21 ..

3 Le Devoir, samedi 22 février 1986. 
relève en s'adjoignant de jeunes collaborateurs, dont Jean-François Chassay qui, avec Sherry Simon, allait lui succéder à la direction.

Reconnue pour l'indépendance de son jugement, elle participa à de nombreux jurys québécois et canadiens et collabora à plusieurs revues québécoises, tant dans le domaine de la littérature que dans celui des arts. Membre du comité directeur de l'édition critique d'Hubert Aquin (ÉDAQ), lectrice aux éditions La Presse, où elle travailla sur le manuscrit de l'Euguélionne, aux Presses de l'Université de Montréal, pour la Fédération canadienne des sciences humaines, et, au moment de sa mort, aux éditions VLB; et enfin chargée de la chronique «la Revue des revues» à Voix \& images, Suzanne Lamy était donc profondément impliquée dans l'institution littéraire québécoise. À Sainte-Adèle, au colloque de l'Académie canadienne-française, elle fit en 1983 une communication intitulée «les Obscures Clartés de la modernité» qui, reliant finement le climat pré- et post-référendaire aux ruptures littéraires de la «nouvelle écriture», témoigne de sa connaissance intime de notre société. De même elle menait, en collaboration avec Jacqueline Chénieux-Gendron du CNRS, une recherche sur les textes québécois marqués par le surréalisme, pour une anthologie qui fera bientôt connaître, dans l'ensemble de la francophonie, tout un pan de notre histoire littéraire, de Refus Global aux poètes regroupés autour de l'Hexagone, et aux romans québécois où l'on retrouve une réminiscence du surréalisme, tels ceux de Ducharme et de Villemaire.

Appartenant à une génération de femmes presque complètement étouffée par la cage, invisible et sans barreaux, du modèle des années 50 , elle a en peu de temps accompli tant de choses, et si bien, que cela reste exemplaire, au-delà de la biographie et de l'anecdote. Avec elle, qui était de plusieurs années mon aînée, je n'ai jamais senti le fossé des gênérations. L'énergie déployée pour utiliser de façon maximale le temps qui lui fut imparti, elle en avait reconnu la puissance dans son insoumission au carcan de la société française, dans son arrachement à l'enfermement des femmes dans un rôle mortifere: elle lui venait d'un instinct de survie qui est, lui, tout à fait québécois. 